\title{
Non-dysplastic ulcerative colitis has high levels of the homologous repair protein NUCKS1 and low levels of the DNA damage marker gamma-H2AX
}

\author{
Paula M. De Angelis, $\mathrm{PhD}^{1}$, Aasa R. Schjølberg ${ }^{1}$, Juliana B. Hughes, $\mathrm{PhD}^{1}$, Henrik S. Huitfeldt, \\ $\mathrm{MD}, \mathrm{PhD}^{2}$, Solveig Norheim Andersen, $\mathrm{MD}, \mathrm{PhD}^{3}$, Anne Carine Østvold, $\mathrm{PhD}^{4}$ \\ ${ }^{1}$ Oslo University Hospital--Rikshospitalet, Dept. of Pathology, Oslo Norway \\ ${ }^{2}$ University of Oslo, Faculty of Medicine, Oslo University Hospital—Rikshospitalet, Dept. of \\ Pathology, Oslo, Norway \\ ${ }^{3}$ Akershus University Hospital, Dept. of Pathology, Lørenskog, Norway \\ ${ }^{4}$ University of Oslo, Institute of Basic Medical Sciences, Dept. of Biochemistry, Oslo, Norway
}

Correspondence to: Paula M. De Angelis, Ph.D., Oslo University Hospital--Rikshospitalet, Dept. of Pathology, Postbox 4950, 0424 Nydalen, Oslo, Norway, Tel: +4723071505

The authors declare that there are no conflicts of interest in this manuscript. 
Background: The colon and rectum are continuously exposed to oxidative stress that generates reactive oxygen species, which are a major cause of DNA double-strand breaks. Furthermore, chronic inflammatory diseases such as ulcerative colitis (UC) are characterized by an excess of reactive nitrogen species that can also lead to DNA double-strand breakage and genomic instability. We investigated the expression of the NUCKS1 (nuclear casein kinase and cyclindependent kinase substrate 1) protein in UC and sporadic colorectal cancer (CRC) due to its involvement in both DNA double-strand break repair and inflammatory signaling. Methods: NUCKS1 expression and expression of the DNA double-strand break marker gamma-H2AX $(\gamma \mathrm{H} 2 \mathrm{AX})$ were assessed in formalin-fixed paraffin-embedded UC and CRC patient biopsies using peroxidase immunohistochemistry. Expression levels for both proteins were evaluated together with previously-published expression level data for hTERT and TP53 proteins in the same material. Results: Non-dysplastic UC lesions had ten-fold lower $\gamma \mathrm{H} 2 \mathrm{AX}$ expression and approximately four-fold higher NUCKS1 expression compared to sporadic CRC, indicating minimal DNA DSB damage and heightened DNA DSB repair in these lesions respectively. NUCKS1 expression in UC tended to decrease with increasing grades of dysplasia, whereas $\gamma \mathrm{H} 2 \mathrm{AX}$, hTERT and TP53 expression tended to increase with increasing grades of dysplasia. The highest $\gamma \mathrm{H} 2 \mathrm{AX}$ expression was seen in sporadic CRC, indicating considerable DNA DSB damage, whereas the highest NUCKS1 and hTERT expression were seen in non-dysplastic UC. Conclusions: Overall, our data suggest that NUCKS1 may be involved in DNA DSB repair and/or inflammatory signaling in UC, but a more thorough investigation of both pathways in UC is warranted. 
Key Words: Ulcerative colitis; sporadic colorectal cancer; NUCKS1; gamma-H2AX ( $\gamma \mathrm{H} 2 \mathrm{AX})$; DNA double-strand breaks; DNA repair protein

5

7

8

9

(1)

.

13

(7)

(9)

.

3

6

(2)

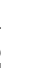

4

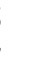

9

.




\section{Introduction}

The colon and rectum are continuously exposed to oxidative stress (1), a cellular condition that favors the production rather than removal of reactive oxygen species (ROS). Cellular ROS are generated via mitochondrial oxidative phosphorylation and NADPH oxidase activity (2) as well as in response to ionizing or ultraviolet radiation, cigarette smoking, alcohol, chronic infections, and inflammatory disorders. ROS play an important role in the progression of inflammatory disorders (3). The chronic inflammation that characterizes the inflammatory bowel disease ulcerative colitis (UC) results from an imbalance of effector lymphocytes and pro-inflammatory cytokines (4). Some of the cytokines, triggered leukocytes and activated macrophages produce large amounts of ROS which can cause oxidative stress. Excessive levels of ROS cause endothelial dysfunction, tissue injury, DNA strand breaks and chromosomal rearrangements $(3,5$, 6). In fact, ROS are a major cause of DNA double-strand breaks (DSB) (7). Chronic inflammation can also lead to an excess of reactive nitrogen species (RNS), which also damage DNA and cause genomic instability $(8,9)$. ROS-induced DNA damage and genomic instabilities (chromosomal and microsatellite) characterize many cancer types $(10,11)$ including sporadic colorectal cancer (CRC). Both ROS- and RNS-induced DNA damage trigger a DNA damage response involving a number of important DNA damage signaling proteins, among them ATM and TP53. The presence of DNA DSB induces the rapid formation of gamma-H2AX $(\gamma \mathrm{H} 2 \mathrm{AX})$ foci (12), making $\gamma \mathrm{H} 2 \mathrm{AX}$ a useful marker of DNA DSB damage (13).

Accurate repair of DNA damage is crucial for the maintenance of genomic stability (14). Repair of DNA DSB is accomplished via homologous recombination (HR) or non-homologous end-joining (NHEJ). The NUCKSI (nuclear casein kinase and cyclin-dependent kinase substrate 1) gene was recently shown to be a paralog of the RAD51API gene that codes for a protein 
involved in HR (15). Depletion of NUCKS1 protein leads to both impaired HR in mammalian cells and increased chromosomal instability, similar to what is seen following RAD51AP1 depletion (15), indicating a role for NUCKS1 in DNA DSB repair and in the maintenance of genomic stability. NUCKS1 silencing also results in reduced activation of the nuclear factor kappa-light-chain-enhancer of activated B cells (NF- $\mathrm{kB})$ protein and reduced expression of the corresponding NFkB-mediated cytokines (16), indicating a role for NUCKS1 in inflammatory signaling. Furthermore, NUCKS1 is involved in chromatin modification, cell death and proliferation signaling $(17,18)$. Overexpression of NUCKS1 has been reported to be an independent prognostic indicator of both poor overall survival and relapse-free survival in CRC (19). There are no reports to date detailing the expression of NUCKS1 in UC. We thus decided to investigate NUCKS1 expression in UC in order to gain a better understanding of its putative role in DNA DSB repair, as this may shed some light on whether DNA DSB repair, specifically HR, is compromised in UC. NUCKS1 expression in sporadic CRC was also evaluated. The $\gamma \mathrm{H} 2 \mathrm{AX}$ protein was used to assess of the presence of DNA DSB. Both NUCKS1 and $\gamma \mathrm{H} 2 \mathrm{AX}$ proteins were examined for possible associations with previously-published expression data for both hTERT (the catalytic subunit of the enzyme telomerase) $(20,21)$ and the tumor suppressor protein TP53 (22). hTERT is of interest because there is a strong association between defective DSB repair, genomic instability, and telomere dysfunction (23), whereas wild-type TP53 plays a major role in DNA damage signaling/repair pathways. 


\section{Materials and Methods}

\section{Ethical Considerations}

Ethical approval to use the UC and sporadic CRC biopsies in this study for research purposes was obtained from the Regional Ethics Committee (REC) of Southeast Norway; the REC \# is 2015/628 (previously S-06062)_Cell biological investigations of neoplasms in the gastrointestinal tract. The ethical approval expires on December $31^{\text {st }} 2025$. The biopsies are included in a research biobank that was approved for research use by the Norwegian Directorate of Health in April 2006. All patient material exists in anonymized form and all data generated from the use of this biobank are stored on a secure hospital server in accordance with government regulations.

\section{Patient material}

\section{$\underline{\text { Ulcerative colitis biopsies }}$}

Colectomy specimens from 13 patients with longstanding UC (10-30 years) resected at the Department of Surgery, Rikshospitalet, in the period 1985-1994 were investigated. These were available as formalin-fixed paraffin-embedded (FFPE) blocks. Biopsy sections were stained with hematoxylin-eosin and evaluated independently by two experienced pathologists according to the classification criteria described by Riddell et al. (24): negative for dysplasia (nondysplastic), indefinite for dysplasia (not possible to classify some epithelial changes as unequivocally positive or negative for dysplasia), and positive for dysplasia (unequivocally neoplastic, designated as low-grade or high-grade based on the degree of deviation from normal 
mucosa). A total of 87 UC biopsies were available for analysis as FFPE material (whole sections and tissue microarray sections), 82 of which had a pathological diagnosis. Fifty-five percent were negative for dysplasia, $11 \%$ were indefinite for dysplasia, $24 \%$ were positive for dysplasia (15\% low-grade, $9 \%$ high-grade), and 10\% were colitis-associated cancer (CAC). All included patients were classified as UC progressors--i.e. cases that had one or more areas of dysplasia or aneuploidy. Seventy-six of 87 UC biopsies had (previously-measured) available DNA ploidy status data; $33 \%$ were aneuploid and $67 \%$ were diploid.

\section{Colorectal cancer biopsies}

Patient material consisted of 57 untreated sporadic colorectal tumor resections taken from patients who had undergone surgery during the period 1990-2001 at three regional hospitals (Ullevål University Hospital, Asker and Bærum County Hospital and Vestfold County hospital). Tumor biopsies were available as FFPE blocks. The patient group consisted of 33 males and 24 females, with a mean age of 69 years. Normal mucosa samples consisted of 24 peritumoral normal mucosas and 14 normal mucosas from colectomy specimens without cancer (designated as non-peritumoral normal mucosa). The degree of tumor spread was assessed using Dukes' modified staging system: 3 tumors were classified as Dukes' A, 31 as Dukes' B, 13 as Dukes' C and 10 as Dukes' D. The majority of this tumor series was previously evaluated for ploidy status (25); 34 of 57 (60\%) tumors were classified as DNA aneuploid and 23 of 57 (40\%) tumors were DNA diploid. 


\begin{abstract}
$\underline{\text { Tissue Microarrays }}$
Preparation of tissue microarrays from formalin-fixed paraffin-embedded (FFPE) colorectal cancer and ulcerative colitis biopsies was described previously in detail (26). The colorectal cancer tissue microarrays utilized in the present study were constructed as follows: 3 cores of tumor tissue from each patient; 2 cores of peritumoral normal mucosa (tissue cores were localized to $<5 \mathrm{~mm}$ from the cancer margin); and 2 cores of non-peritumoral normal mucosa.
\end{abstract}

\title{
Immunohistochemistry for NUCKS1 protein
}

The immunohistochemical staining method for the NUCKS1 protein has been described in an earlier publication (27). FFPE tissue microarray sections and whole sections of CRC and UC biopsies were immunohistochemically stained for the NUCKS1 protein. Four micron thick tissue sections were deparaffinized, rehydrated and treated with 3\% hydrogen peroxide for 30 min to block endogenous peroxidase, and washed in double-distilled water three times for $5 \mathrm{~min}$ each. Antigen retrieval was accomplished using a trypsin solution $\left(0.1 \%\right.$ trypsin in $0.1 \% \mathrm{CaCl}_{2}$, $20 \mathrm{mM}$ Tris- $\mathrm{HCl}, \mathrm{pH} 7.8$ ) applied to the slides for $10 \mathrm{~min}$ at $37^{\circ} \mathrm{C}$. Slides were then rinsed in tap water for 5 min followed by immersion in double-distilled water three times for 5 min each and in PBS (pH 6.09) 3 times for 5 min each. Slides were then incubated in blocking solution (PBS containing $1 \%$ BSA and 5\% normal goat serum) for 1 hour at room temperature. Affinitypurified goat anti-rabbit anti-NUCKS1 antibody (28) was made up in the same blocking solution at a 1:300 dilution and applied to the slides. The slides were covered with plastic cover slips and incubated overnight at $4^{\circ} \mathrm{C}$ in a humidity chamber. Slides were then rinsed three times in PBS for 5 min each time and incubated with ImmPress Anti-Rabbit Ig Peroxidase (Vector Laboratories, 
Burlingame CA) for 30 min at room temperature, followed by washing three times in PBS for 5 min each time and visualization with DAB Substrate Kit for Peroxidase (Vector Laboratories, Burlingame CA) for 10-15 min at room temperature. The reaction was stopped by placing slides in double-distilled water for $5 \mathrm{~min}$, and then the slides were counterstained with hematoxylin, washed and mounted. Tissue sections where the primary antibody was replaced with blocking solution served as negative staining controls. Stained slides were evaluated for staining quality and specificity; positive (brown) nuclei were counted. Three to four hundred epithelial (UC, $\mathrm{CRC}$ and normal colonic mucosa) cells per tissue microarray core were counted for each case for a total of circa 1000 cells per patient. Likewise, several areas were counted on the whole sections for a total of circa 1000 cells per patient. NUCKS1 expression levels were defined as the percentage of positively-stained nuclei out of 1000 total cells counted.

\section{Immunohistochemistry for $\gamma H 2 A X$ protein}

FFPE tissue microarray sections and whole sections of CRC and UC biopsies were immunohistochemically stained for the $\gamma \mathrm{H} 2 \mathrm{AX}$ protein. Slides were deparaffinized, rehydrated and treated with $3 \%$ hydrogen peroxide for 30 min to block endogenous peroxidase, washed in double-distilled water three times for $5 \mathrm{~min}$ each, and subjected to microwave antigen retrieval in citrate buffer $\mathrm{pH}$ 6.0. Cooled slides were incubated in blocking solution (TBS containing 3\% BSA) for 30 min at room temperature. Mouse monoclonal anti-gamma-H2A.X antibody (Ser139,

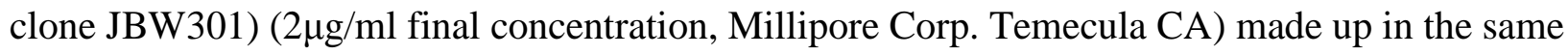
blocking solution was added to the slides that were then covered with plastic cover slips and incubated overnight at $4^{\circ} \mathrm{C}$ in a humidity chamber. Slides were rinsed three times in PBS for 5 
min each time, and then incubated with ImmPress Anti-Mouse Ig Peroxidase (Vector Laboratories, Burlingame CA) for $30 \mathrm{~min}$ at room temperature. Slides were then washed three times in PBS for 5 min each time and then visualized with DAB Substrate Kit for Peroxidase (Vector Laboratories, Burlingame CA) for 10-15 min at room temperature. The reaction was stopped by placing slides in double-distilled water for $5 \mathrm{~min}$, and then the slides were counterstained with hematoxylin, washed and mounted. Tissue sections where the primary antibody was replaced with blocking solution served as negative staining controls. Brownstained nuclei were counted as positive. Three to four hundred cells per tissue microarray core were counted for each case for a total of circa 1000 cells per patient. Likewise, several areas were counted on the whole sections for a total of circa 1000 cells per patient. $\gamma \mathrm{H} 2 \mathrm{AX}$ expression levels were defined as the percentage of positively-stained nuclei out of 1000 total cells counted.

\section{Slide scanning}

Stained slides were digitally scanned using a bright-field Pannoramic MIDI scanner with a 20X objective from 3DHistech (Budapest, Hungary) and image preparation was done using the 3DHistech Pannoramic Viewer software.

\section{Statistical analyses}

GraphPad Prism version 7 (GraphPad Software, La Jolla, CA, USA) was used for statistical analyses. Frequency distributions for protein expressions in the various tissue groups were used to determine whether the mean (parametric distribution) or the median was to be used as the cut-off level of expression for statistical testing and reporting. The relationships between 
protein expression levels and clinicopathological features (e.g. gender, age, diagnosis, and tumor site) were evaluated using contingency testing and correlation analyses; in UC, since each patient contributed with multiple biopsies with the same gender and age information, levels of protein expression were evaluated in relation to the pathological diagnosis alone. A p-value $<0.05$ denoted statistical significance.

\section{Results \\ NUCKS1 and $\gamma H 2 A X$ expression in UC, CRC, and normal colonic mucosa}

Representative images of UC, CRC, and normal colonic mucosal tissues immunohistochemically stained for NUCKS1 are shown in Figure 1A-C. The NUCKS1 protein was localized predominantly to the nucleus in epithelial cells, but cytoplasmic staining was also seen in a small number of cells. The pattern and intensity of staining were fairly homogeneous and strong, respectively. Representative images of UC, CRC, and normal colonic mucosal tissues immunohistochemically stained for $\gamma \mathrm{H} 2 \mathrm{AX}$ are shown in Figure 2A-C. The $\gamma \mathrm{H} 2 \mathrm{AX}$ protein was localized to the nucleus, and the pattern and intensity of staining were homogeneous and strong, respectively.

All 65 UC lesions were positive for NUCKS1 and expression levels ranged from 4 to 94\%. Non-dysplastic UC lesions had the highest NUCKS1 expression (median of 51\%, range 14 to $94 \%$ ), and indefinite for dysplasia UC lesions had the lowest (median of 10\%, range 4 to $78 \%$ ), but this difference was not significant. Fifty-two of 53 (98\%) sporadic CRC lesions showed positive NUCKS1 staining; expression levels ranged from 0 to $67 \%$ (median of 13\%). Thirty- 
three of 37 (89\%) normal mucosas were positive for NUCKS1, with expression levels ranging from 0 to $67 \%$ (median of $15 \%$ ). Significant differences in NUCKS1 expression were seen between UC non-dysplasia and sporadic CRC ( $\mathrm{p}<0.001)$ and UC non-dysplasia and peritumoral normal colonic mucosa (median of 14\%, range 0 to 54\%) (p < 0.001) (Figure 3A). NUCKS1 expression in peritumoral normal colonic mucosa was significantly lower than in nonperitumoral colonic mucosa (median of $26 \%$, range 7 to $67 \%)(\mathrm{p}<0.05)$ (Figure $3 \mathrm{~A})$.

Forty-three of $70(61 \%)$ UC lesions were positive for $\gamma \mathrm{H} 2 \mathrm{AX}$ and expression levels ranged from 0 to $40 \%$ (median of $2 \%$ ). All sporadic CRC cases were positive for $\gamma \mathrm{H} 2 \mathrm{AX}$ with expression levels ranging from 2 to $73 \%$ (median of 22\%). Thirty-seven of 38 (97\%) normal mucosas expressed $\gamma \mathrm{H} 2 \mathrm{AX}$, and expression levels ranged from 0 to $9 \%$ (median of $4 \%$ ). Thus the highest $\gamma \mathrm{H} 2 \mathrm{AX}$ expression was seen in sporadic $\mathrm{CRC}$ (Figure 3B). $\gamma \mathrm{H} 2 \mathrm{AX}$ expression was low in all UC lesions, but lowest in non-dysplastic lesions (median 0\%, range 0 to 13\%) (Figure 3B); differences among the UC groups were not significant. Significant differences in $\gamma \mathrm{H} 2 \mathrm{AX}$ expression were seen between sporadic CRC and non-dysplastic UC lesions $(\mathrm{p}<0.001)$, sporadic CRC and dysplasia ( $\mathrm{p}<0.01)$, and sporadic CRC and indefinite for dysplasia $(\mathrm{p}<0.01)$. Peritumoral and non-peritumoral normal mucosa had similar $\gamma \mathrm{H} 2 \mathrm{AX}$ expression that was significantly lower than $\gamma \mathrm{H} 2 \mathrm{AX}$ expression in sporadic CRC ( $\mathrm{p}<0.001$ for both).

NUCKS1 and $\gamma H 2 A X$ levels in relation to clinicopathological parameters, ploidy status, hTERT expression and TP53 expression

Since each UC patient contributed with multiple biopsies with the same gender and age information, expressions of NUCKS1 and $\gamma \mathrm{H} 2 \mathrm{AX}$ were evaluated in relation to UC morphology 
alone and not other clinicopathological parameters. We found no significant correlations of NUCKS1 or $\gamma \mathrm{H} 2 \mathrm{AX}$ with ploidy status in UC. There were no significant associations or observed trends in the expression of NUCKS1 or $\gamma \mathrm{H} 2 \mathrm{AX}$ in sporadic CRC when evaluated in relation to gender, age, Dukes' stage, tumor site, or tumor ploidy status.

In UC, NUCKS1 was significantly inversely correlated with $\gamma \mathrm{H} 2 \mathrm{AX}$ expression $(\mathrm{r}=$ $0.29, \mathrm{p}=0.04)$. Expression levels for NUCKS1 and $\gamma \mathrm{H} 2 \mathrm{AX}$ in UC were also evaluated together with previously-published expression level data for hTERT and TP53 proteins in UC $(20,22)$. The majority of UC lesions except for CAC did not express TP53 (Figure 3C and (22)). hTERT levels increased with increasing grades of dysplasia (Figure 3D and (20). NUCKS1 was significantly inversely correlated with hTERT expression $(r=-0.42, p=0.002) \cdot \gamma \mathrm{H} 2 \mathrm{AX}$ expression was significantly positively correlated with hTERT expression $(r=0.47, p=0.0005)$. $\gamma \mathrm{H} 2 \mathrm{AX}$ expression was significantly positively correlated with TP53 expression $(\mathrm{r}=0.3, \mathrm{p}=$ 0.03), as was hTERT expression $(\mathrm{r}=0.34, \mathrm{p}=0.02)$.

NUCKS1 expression was significantly inversely correlated with $\gamma \mathrm{H} 2 \mathrm{AX}$ expression in both sporadic CRC $(r=-0.36, p=0.008)$ and normal colonic mucosa $(r=-0.35, p=0.04)$. Expression levels for NUCKS1 and $\gamma \mathrm{H} 2 \mathrm{AX}$ in sporadic CRC and normal colonic mucosa were also evaluated together with previously-published expression level data for hTERT in sporadic CRC (21). hTERT levels were higher in the CRC group compared to normal mucosas (Figure 3D and (21), but lower overall compared to UC. In normal mucosa, $\gamma \mathrm{H} 2 \mathrm{AX}$ was significantly inversely correlated with hTERT expression $(r=-0.51, \mathrm{p}=0.0013)$, whereas NUCKS1 was positively correlated with hTERT expression $(\mathrm{r}=0.4, \mathrm{p}=0.01)$. 


\section{Discussion}

The multi-functional NUCKS1 protein is reported to play a role in both DNA DSB damage repair and in inflammation signaling. Although it is well-known that chronic inflammation and oxidative stress characterize UC, little is known about DNA DSB repair in the UC colon. Other DNA repair processes in UC, e.g. mismatch repair, have been characterized previously $(29,30)$. The negative for dysplasia UC classification is especially interesting in the context of DNA damage/repair because this group includes all inflammatory and regenerative lesions that undergo recurrent episodes of ulceration and repair (24). The DNA damage caused by oxidative stress in the damage-regeneration cycle is thought to be one of the contributing factors in the development of CRC in UC patients (30). Non-dysplastic UC lesions had nearly four-fold higher NUCKS1 expression compared to sporadic CRC, likely reflecting heightened DNA DSB repair in response to oxidative and nitrative stresses. NUCKS1 happens to be one of many proteins that is a substrate for the ATM kinase in vitro and in vivo (31). ATM is activated in response to DNA DSB, whereby it subsequently activates the DNA damage response pathway. Interestingly, NUCKS1 is phosphorylated by ATM in response to ionizing radiation-induced DNA DSB and oxidative stress (32). The role of NUCKS1 in DNA DSB repair in non-dysplastic UC does not preclude the involvement of other DNA DSB repair proteins, and this will be investigated further. The tenfold lower $\gamma \mathrm{H} 2 \mathrm{AX}$ expression in non-dysplastic UC (compared to sporadic CRC) indicates a low level of DNA DSB that may be the result of effective repair of oxidative stress-induced DNA DSB in UC, or alternatively that DNA DSB damage/genomic instability are less frequent in UC compared to CRC. Effective repair of oxidative stress-induced DNA DSB is in line with (15) who suggested that elevated NUCKS1 levels could reduce the consequences of replication stress that lead to DSB and genomic instability and thus provide a selective advantage for precancerous cells. If however DNA DSB damage/genomic instability 
are less frequent in UC compared to CRC, high NUCKS1 expression in non-dysplastic UC may point to an association with signaling pathways other than DNA repair, e.g. inflammation. An association of NUCKS1 with inflammation in UC is plausible since the UC non-dysplasia group includes all inflammatory and regenerative lesions that undergo continual tissue destruction and regeneration. Moreover, NUCKS1 is known to activate the multifunctional NF-kB protein and the corresponding NFKB-mediated cytokines (16). Since ROS challenge the entire UC colon (33), it doesn't seem likely that non-dysplastic UC lesions would experience different levels of ROSinduced replication stress relative to the other lesions. However, levels of active inflammation could be different among the non-dysplasia, indefinite for dysplasia and positive for dysplasia UC groups, but this is not known at present, and we have not investigated the possible differences. Then again, NUCKS1 could be involved in both DNA DSB repair and inflammatory signaling in non-dysplastic UC. NUCKS1 levels in non-peritumoral colonic mucosa most likely reflect the normal expression of NUCKS1 in response to both oxidative and nitrative stresses in the colon, whereas the lower NUCKS1 levels in peritumoral colonic mucosa could be explained by the influence of sporadic CRC field effects on the peritumoral mucosa $(34,35)$.

The higher $\gamma \mathrm{H} 2 \mathrm{AX}$ expression in sporadic CRC compared to UC indicates that DNA DSB damage/genomic instability are more extensive in CRC than in UC generally, a plausible scenario given the widespread chromosomal instability (CIN) that characterizes a majority of sporadic CRC $(25,36)$. CIN, an increased rate of chromosome missegregation in mitosis that leads to gross chromosomal aneuploidy (37), is a hallmark of many cancers and is associated with a lower expression of DNA repair and chromatin maintenance genes (38). This could explain the lower levels of NUCKS1 in sporadic CRC compared to UC. The incidence of aneuploidy in sporadic CRC was approximately double that seen in UC (60\% versus $33 \%$ 
respectively) in the present study, consistent with a higher CIN activity in this group compared to the UC lesions where microsatellite instability dominates.

Some $\gamma \mathrm{H} 2 \mathrm{AX}$ expression in CRC and UC could be explained by apoptosis, since doublestranded DNA apoptotic fragments are labeled by the $\gamma \mathrm{H} 2 \mathrm{AX}$ antibody (39). The high $\gamma \mathrm{H} 2 \mathrm{AX}$ expression seen for sporadic CRC in the present study is mainly due to DNA damage and not apoptosis, since levels of natural apoptosis in sporadic CRC range from 0 to $5 \%(40,41)$. In contrast, $\gamma \mathrm{H} 2 \mathrm{AX}$ expression in UC may be due to apoptosis, since apoptotic levels in active UC have been reported to vary from $2.5-3 \%$ (42), in line with the $2 \%$ median $\gamma \mathrm{H} 2 \mathrm{AX}$ expression level in our UC lesions. The positive correlation of $\gamma \mathrm{H} 2 \mathrm{AX}$ and hTERT expression in UC lends support to the findings of di Fagagna et al. (43) who reported that telomere shortening triggers a DNA damage response involving H2AX phosphorylation at telomeres. hTERT levels were quite high in UC generally, consistent with its role in compensating for the telomere loss that occurs due to the telomere shortening that is characteristic of UC (20).

Interestingly, expression levels of NUCKS1, $\gamma \mathrm{H} 2 \mathrm{AX}$, and hTERT tended to differ between $\mathrm{CAC}$ and sporadic CRC. These observations are in agreement with reports showing that CAC and sporadic CRC appear to be two biologically different entities based on differences in the spectrum of genomic alterations that characterize them individually $(30,44)$.

Since chronic inflammation and DNA damage characterize UC, one might expect to detect wild-type TP53 expression (especially in the non-dysplastic UC group) given the role of TP53 in DNA damage signaling and in the suppression of inflammation, but this was not the case. It could be speculated that the negligible TP53 expression seen in the non-dysplastic UC group is due to suppression of TP53 by factors that are known to suppress TP53, e.g. NF-кB (45, 
46), but further studies are necessary to determine whether this is the case. For example,

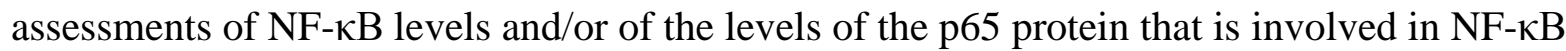
heterodimer formation, nuclear translocation and activation could help to answer this question, and will be the focus of future studies.

Overall, our data suggest possible roles for NUCKS1 in DNA DSB repair and/or inflammatory signaling in UC, but a more thorough investigation of both pathways in UC is warranted. Investigation of the expression of other DNA DSB repair proteins and of inflammation-associated proteins will provide a more complete picture of the putative relationship between DSB repair and inflammatory signaling in both UC and CRC. Future studies will explore these scenarios and are already underway.

\section{Acknowledgments}

This work was supported by the Astri and Birger Torsteds Foundation.

\section{References}

1. Blau S, Rubinstein A, Bass P, et al. Differences in the reducing power along the rat GI tract: Lower antioxidant capacity of the colon. Mol Cell Biochem. 1999;194:185-191

2. Panday A, Sahoo MK, Osorio D, et al. NADPH oxidases: an overview from structure to innate immunity-associated pathologies. Cell Mol Immunol. 2014;12:5-23

3. Mittal M, Siddiqui MR, Tran K, et al. Reactive Oxygen Species in Inflammation and Tissue Injury. Antioxid Redox Signal. 2014;20:1126-1167 
4. Pereira C, Coelho R, Grácio D, et al. DNA Damage and Oxidative DNA Damage in Inflammatory Bowel Disease. J Crohns Colitis. 2016;10:1316-1323

5. Valko M, Izakovic M, Mazur M, et al. Role of oxygen radicals in DNA damage and cancer incidence. Mol Cell Biochem. 2004;266:37-56

6. Valko M, Rhodes CJ, Moncol J, et al. Free radicals, metals and antioxidants in oxidative stress-induced cancer. Chem Biol Interact. 2006;160:1-40

7. Lieber M. The Mechanism of Double-Strand DNA Break Repair by the Nonhomologous DNA End-Joining Pathway. Annu Rev Biochem. 2010;79:181-211

8. Sawa T, Ohshima H. Nitrative DNA damage in inflammation and its possible role in carcinogenesis. Nitric Oxide. 2006;14:91-100

9. Murata M, Thanan R, Ma N, et al. Role of Nitrative and Oxidative DNA Damage in Inflammation-Related Carcinogenesis. J Biomed Biotechnol. 2012;2012

10. Limoli CL, Giedzinski E. Induction of Chromosomal Instability by Chronic Oxidative Stress. Neoplasia (New York, NY). 2003;5:339-346

11. Maneval ML, Eckert KA. Effects of oxidative and alkylating damage on microsatellite instability in nontumorigenic human cells. Mutation Research/Fundamental and Molecular Mechanisms of Mutagenesis. 2004;546:29-38

12. Rogakou EP, Pilch DR, Orr AH, et al. DNA Double-stranded Breaks Induce Histone H2AX Phosphorylation on Serine 139. J Biol Chem. 1998;273:5858-5868

13. McManus K, Hendzel M. ATM-dependent DNA damage-independent mitotic phosphorylation of H2AX in normally growing mammalian cells. Mol Biol Cell. 2005;16:50135025

14. Shen Z. Genomic instability and cancer: an introduction. J Mol Cell Biol. 2011;3:1-3 
15. Parplys AC, Zhao W, Sharma N, et al. NUCKS1 is a novel RAD51AP1 paralog important for homologous recombination and genome stability. Nucleic Acids Res. $2015 ; 43: 9817-9834$

16. Poon M-W, Jiang D, Qin P, et al. Inhibition of NUCKS Facilitates Corneal Recovery Following Alkali Burn. Scientific Reports. 2017;7:41224

17. Qiu B, Shi X, Wong ET, et al. NUCKS is a positive transcriptional regulator of insulin signaling. Cell Rep. 2014;7:1876-1886

18. Qiu B, Shi X, Zhou Q, et al. Hypothalamic NUCKS regulates peripheral glucose homoeostasis. Biochem J. 2015;469:391-398

19. Kikuchi A, Ishikawa T, Mogushi K, et al. Identification of NUCKS1 as a colorectal cancer prognostic marker through integrated expression and copy number analysis. Int J Cancer. $2013 ; 132: 2295-2302$

20. Friis-Ottessen M, De Angelis PM, Schjolberg AR, et al. Reduced hTERT protein levels are associated with DNA aneuploidy in the colonic mucosa of patients suffering from longstanding ulcerative colitis. Int J Mol Med. 2014;33:1477-1483

21. Schjolberg AR, Clausen OP, Burum-Auensen E, et al. Aneuploidy is associated with TP53 expression but not with BRCA1 or TERT expression in sporadic colorectal cancer. Anticancer Res. 2009;29:4381-4387

22. Friis-Ottessen M, Burum-Auensen E, Schjølberg A, et al. TP53/p53 alterations and Aurora A expression in progressor and non-progressor colectomies from patients with longstanding ulcerative colitis. Int J Mol Med. 2015;35:24-30

23. Sharma GG, Gupta A, Wang H, et al. hTERT associates with human telomeres and enhances genomic stability and DNA repair. Oncogene. 2003;22:131-146 
24. Riddell R, Goldman H, Ransohoff D, et al. Dysplasia in inflammatory bowel disease: standardized classification with provisional clinical applications. Hum Pathol. 1983;14:931-968 25. De Angelis PM, Clausen OPF, Schjolberg A, et al. Chromosomal gains and losses in primary colorectal carcinomas detected by $\mathrm{CGH}$ and their associations with tumour DNA ploidy, genotypes and phenotypes. Br J Cancer. 1999;80:526-535

26. Burum-Auensen E, DeAngelis PM, Schjoelberg AR, et al. Spindle proteins Aurora A and BUB1B, but not Mad2, are aberrantly expressed in dysplastic mucosa of patients with longstanding ulcerative colitis. J Clin Pathol 2007;60:1403-1408

27. Drosos Y, Kouloukoussa M, Ostvold AC, et al. NUCKS overexpression in breast cancer. Cancer Cell Int. 2009;9:19

28. Østvold AC, Norum JH, Mathiesen S, et al. Molecular cloning of a mammalian nuclear phosphoprotein NUCKS, which serves as a substrate for Cdk1 in vivo. Eur J Biochem. 2001;268:2430-2440

29. Pokorny RM, Hofmeister A, Galandiuk S, et al. Crohn's disease and ulcerative colitis are associated with the DNA repair gene MLH1. Annals of Surgery. 1997;225:718-725

30. Seril DN, Liao J, Yang G-Y, et al. Oxidative stress and ulcerative colitis-associated carcinogenesis: studies in humans and animal models. Carcinogenesis. 2003;24:353-362

31. Grundt K, Thiede B, Østvold AC. Identification of kinases phosphorylating 13 sites in the nuclear, DNA-binding protein NUCKS. Biochimica et Biophysica Acta (BBA) - Proteins and Proteomics. 2017;1865:359-369

32. Matsuoka S, Ballif BA, Smogorzewska A, et al. ATM and ATR substrate analysis reveals extensive protein networks responsive to DNA damage. Science. 2007;316:1160-1166 
33. Rogler G. Chronic ulcerative colitis and colorectal cancer. Cancer Letters. 2014;345:235241

34. Bernstein C, Bernstein H, Payne CM, et al. Field defects in progression to gastrointestinal tract cancers. Cancer Letters. 2008;260:1-10

35. Hawthorn L, Lan L, Mojica W. Evidence for field effect cancerization in colorectal cancer. Genomics. 2014;103:211-221

36. Ried T, Knutzen R, Steinbeck R, et al. Comparative genomic hybridization reveals a specific pattern of chromosomal gains and losses during the genesis of colorectal tumors. Genes Chromosomes Cancer. 1996;15:234-245

37. Yuen KWY. Chromosome Instability (CIN), Aneuploidy and Cancer. eLS: John Wiley \& Sons, Ltd; 2001

38. Roschke A, Glebov O, Lababidi S, et al. Chromosomal Instability Is Associated with Higher Expression of Genes Implicated in Epithelial-Mesenchymal Transition, Cancer Invasiveness, and Metastasis and with Lower Expression of Genes Involved in Cell Cycle Checkpoints, DNA Repair, and Chromatin Maintenance. Neoplasia. 2008;10:1222-1230 39. Rogakou EP, Nieves-Neira W, Boon C, et al. Initiation of DNA Fragmentation during Apoptosis Induces Phosphorylation of H2AX Histone at Serine 139. J Biol Chem. 2000;275:9390-9395

40. De Angelis PM, Stokke T, Thorstensen L, et al. Apoptosis and expression of Bax, Bcl-x, and Bcl-2 apoptotic regulatory proteins in colorectal carcinomas, and association with p53 genotype/phenotype. Molecular Pathology. 1998;51:254-261

41. Sinicrope FA, Roddey G, McDonnell TJ, et al. Increased apoptosis accompanies neoplastic development in the human colorectum. Clin Cancer Res. 1996;2:1999-2006 
42. Iwamoto M, Takehiko K, Makiyama K, et al. APOPTOSIS OF CRYPT EPITHELIAL CELLS IN ULCERATIVE COLITIS. J Pathol. 1996;180:152-159

43. di Fagagna FD, Reaper PM, Clay-Farrace L, et al. A DNA damage checkpoint response in telomere-initiated senescence. Nature. 2003;426:194-198

44. Yaeger R, Shah MA, Miller VA, et al. Genomic Alterations Observed in ColitisAssociated Cancers Are Distinct From Those Found in Sporadic Colorectal Cancers and Vary by Type of Inflammatory Bowel Disease. Gastroenterology. 2016;151:278-287.e276

45. Gudkov AV, Gurova KV, Komarova EA. Inflammation and p53: A Tale of Two Stresses. Genes \& Cancer. 2011;2:503-516

46. Gudkov AV, Komarova EA. p53 and the Carcinogenicity of Chronic Inflammation. Cold Spring Harb Perspect Med. 2016;6:a026161

\section{Figure legends}

Figure 1. NUCKS1 expression in UC, CRC, and normal colonic mucosa

Formalin-fixed sections evaluated immunohistochemically for NUCKS1: A) in a non-dysplastic UC lesion; B) in a sporadic CRC; C) in a normal colonic mucosa. 200X magnification. Scale bar $=100 \mu \mathrm{m}$.

\section{Figure 2. $\gamma \mathrm{H} 2 \mathrm{AX}$ expression in $\mathrm{UC}, \mathrm{CRC}$, and normal colonic mucosa} Formalin-fixed sections evaluated immunohistochemically for $\gamma \mathrm{H} 2 \mathrm{AX}$ : A) in a low-grade dysplastic UC lesion; B) in a sporadic CRC; C) in a normal colonic mucosa. 200X magnification. Scale bar $=100 \mu \mathrm{m}$.

Figure 3. NUCKS1, $\gamma \mathrm{H} 2 \mathrm{AX}$, TP53 and hTERT expression levels in UC, sporadic CRC and normal colonic mucosa. The box and whiskers plots in figures A-D show the median levels of protein expression as horizontal lines inside the boxes. The boxes themselves indicate the ranges of expression levels for each protein. Expression level data for TP53 and hTERT were from our previously-published reports (20-22). 


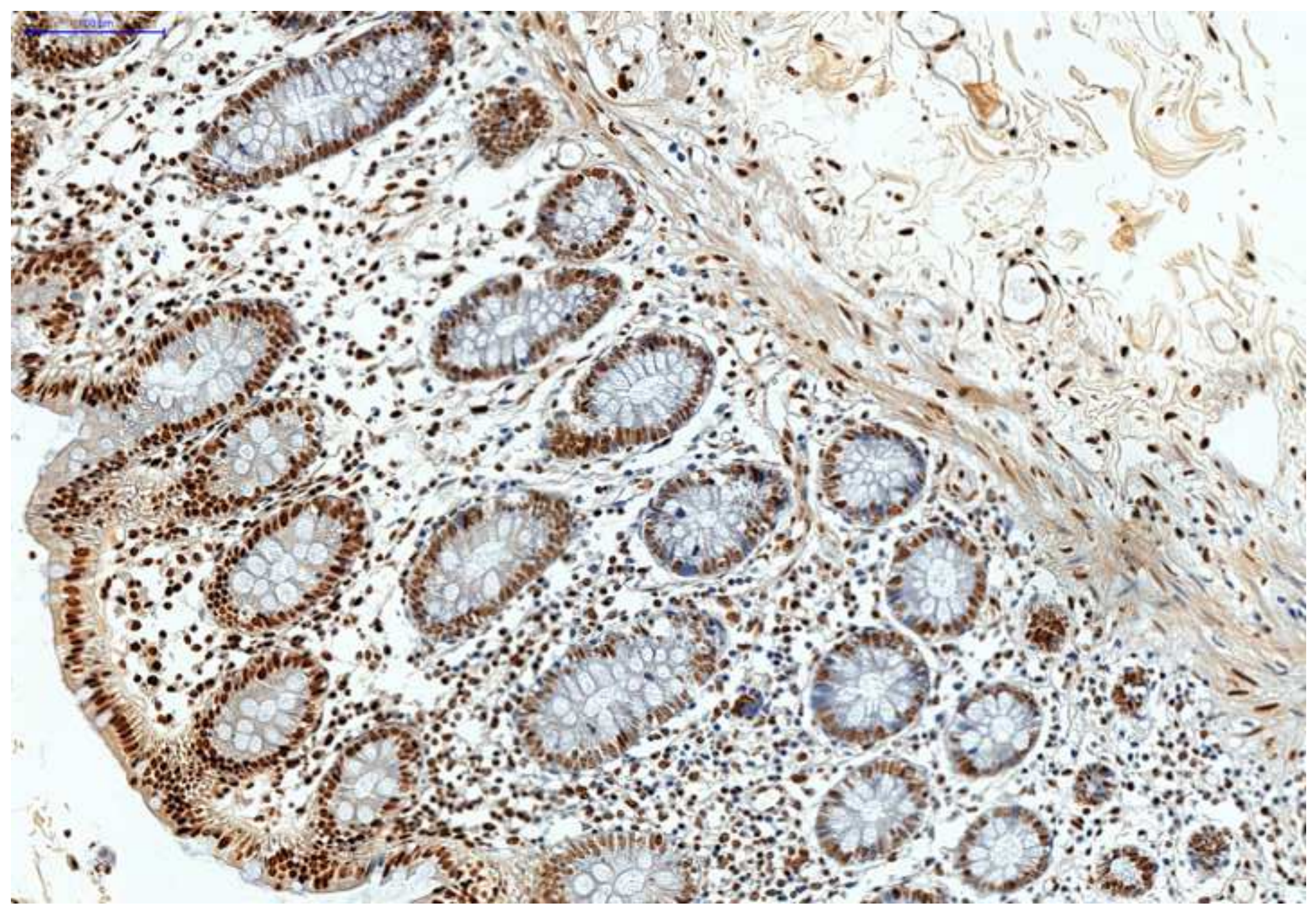




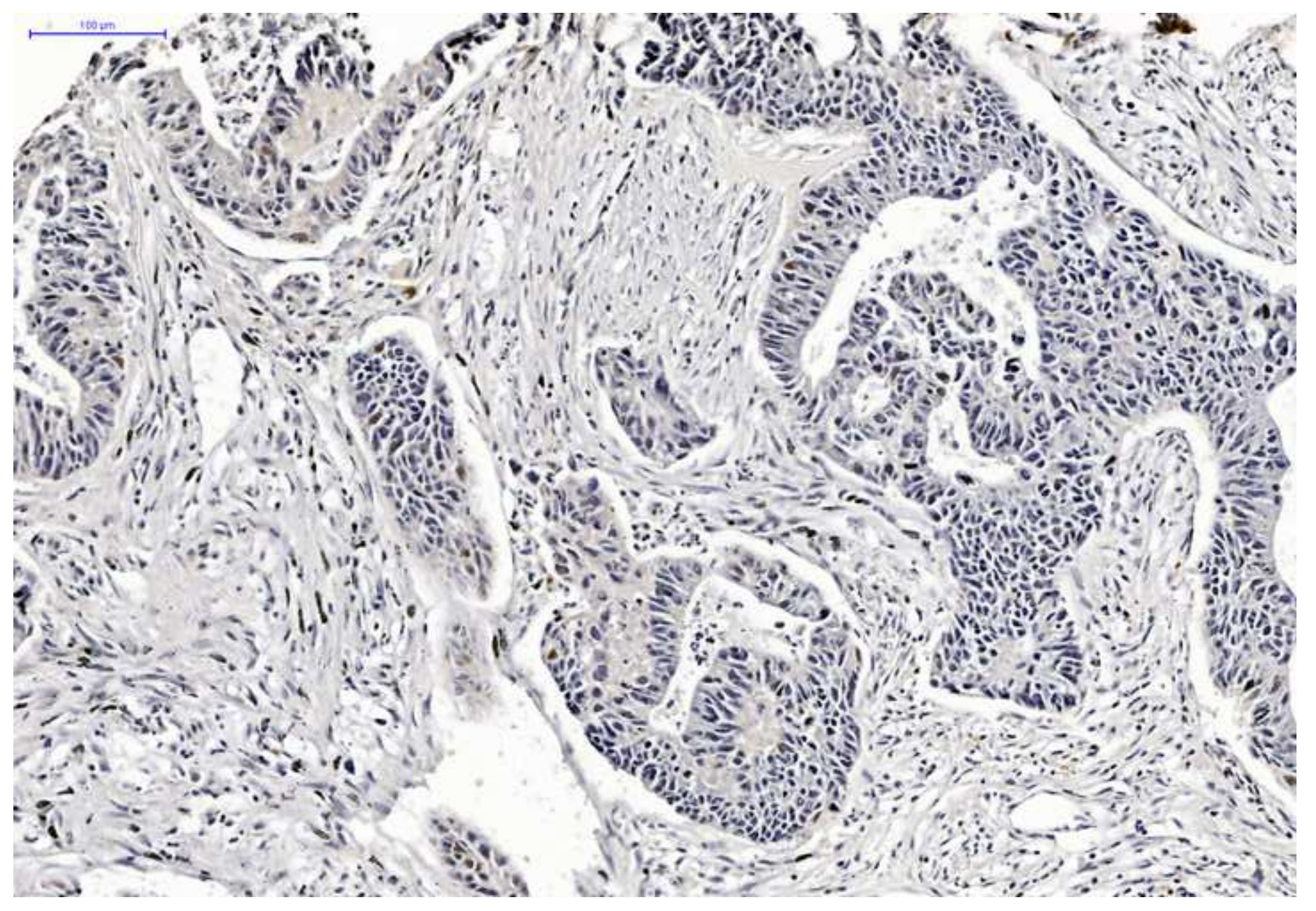




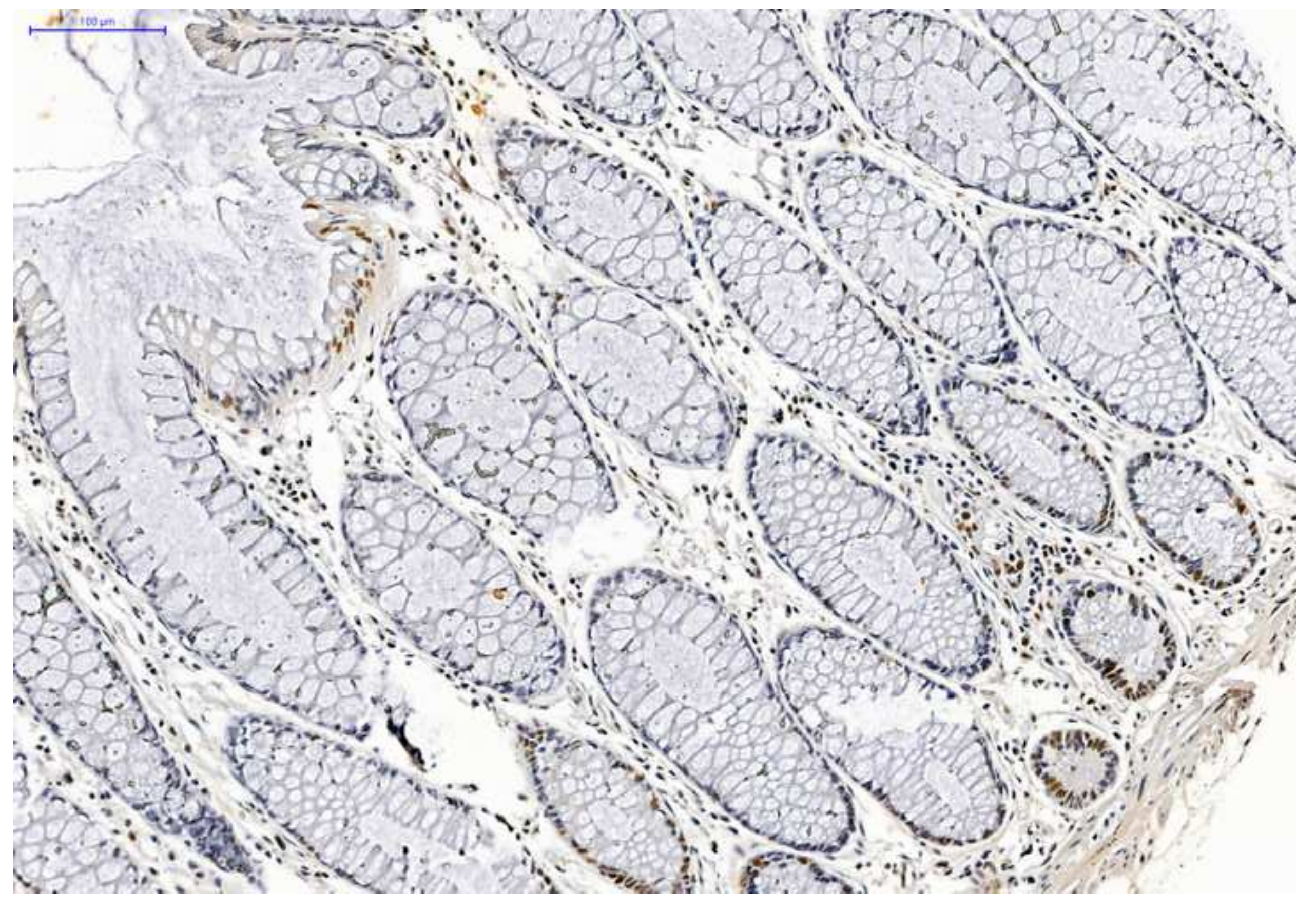




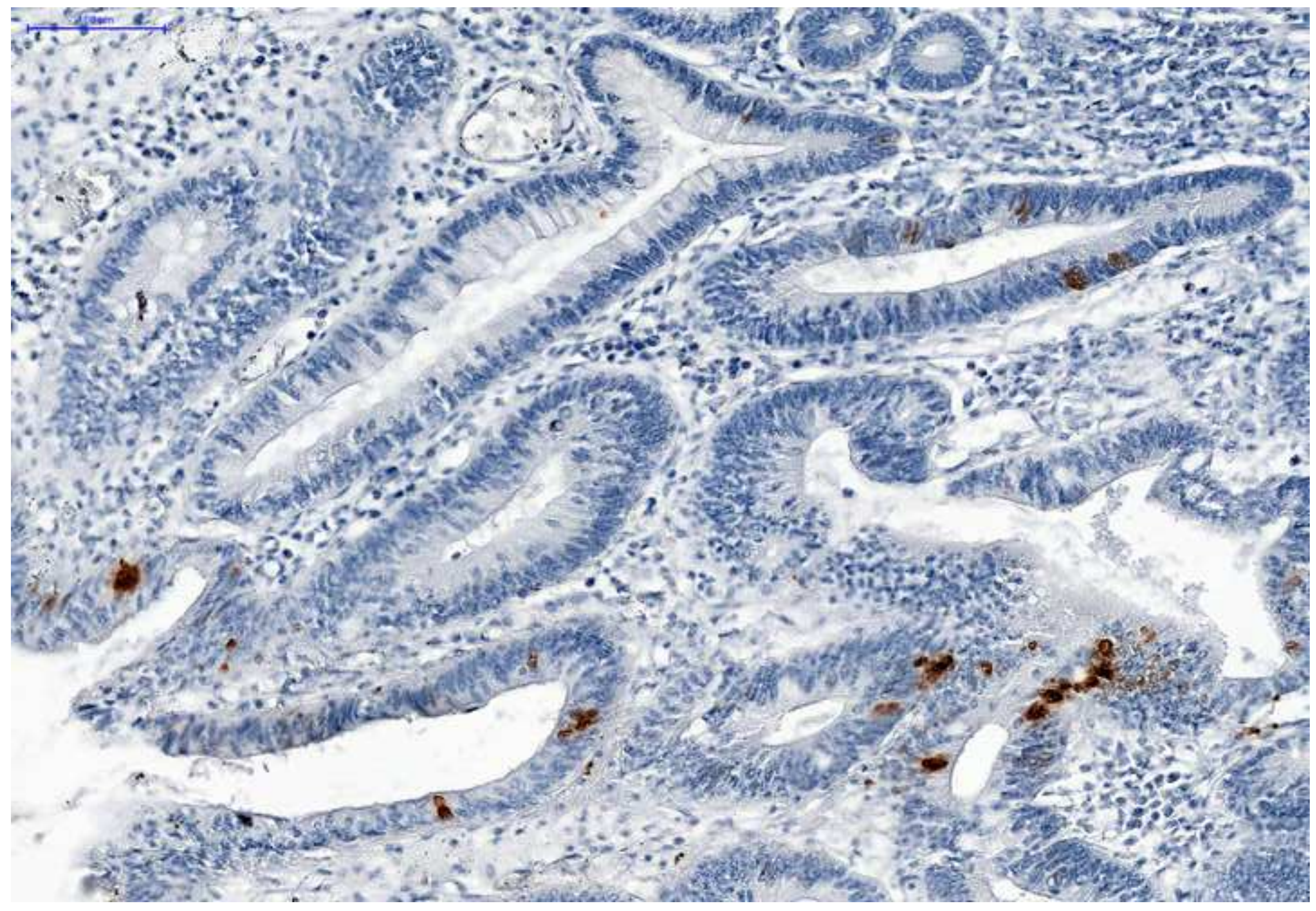




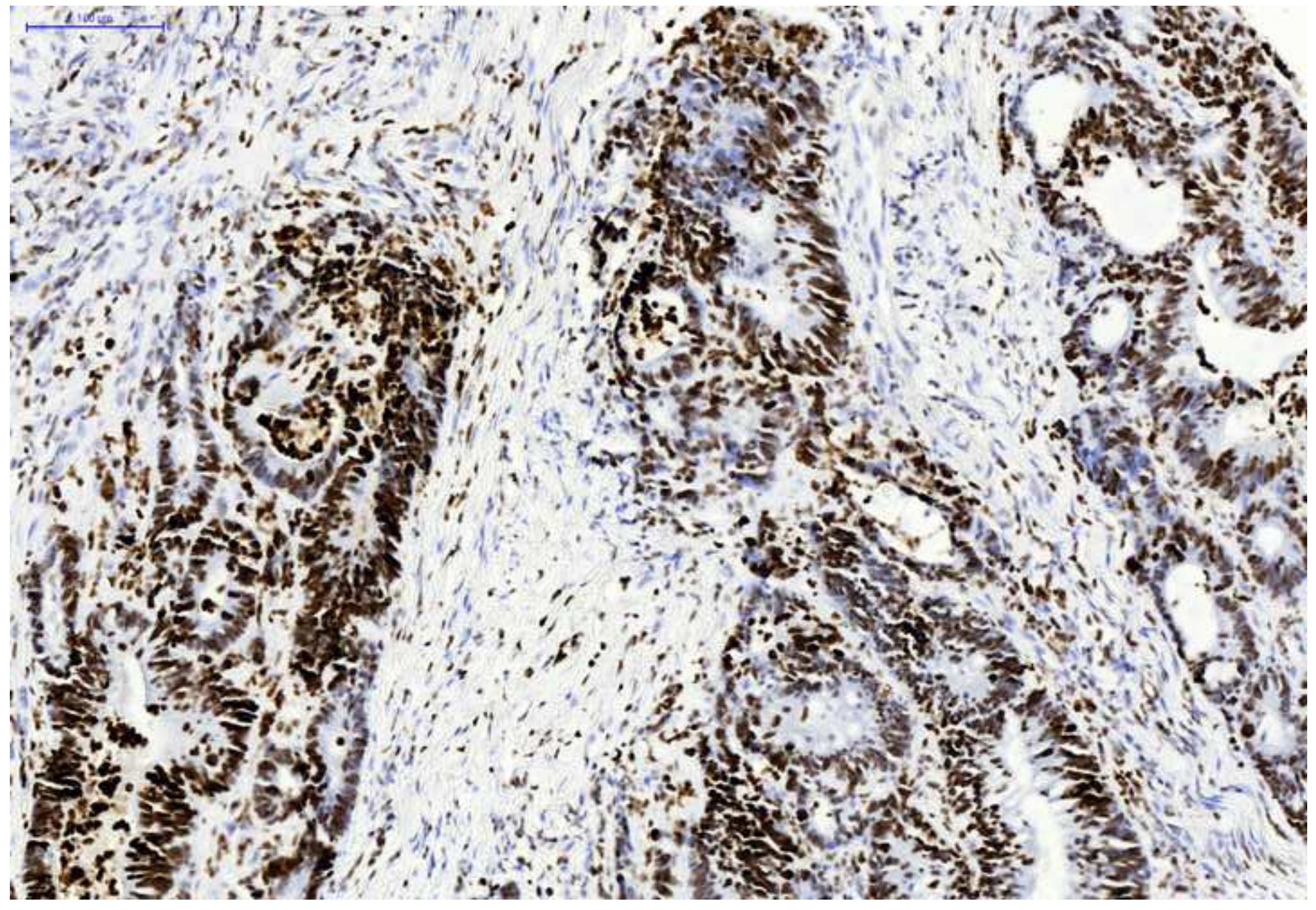




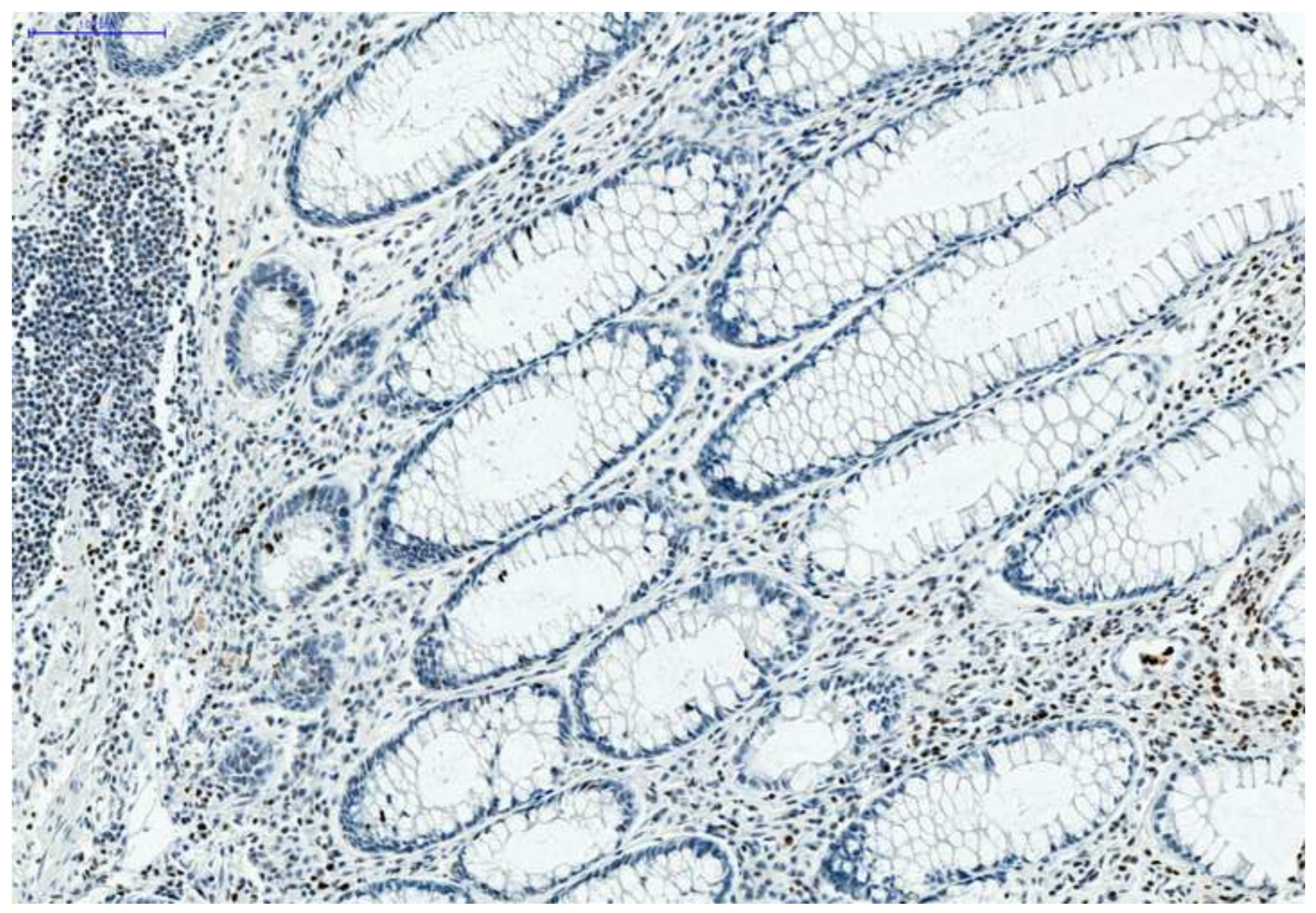


Figure 3. NUCKS1, $\gamma \mathrm{H} 2 \mathrm{AX}$, TP53 and hTERT expression in ulcerative colitis, colorectal cancer and normal colonic mucosa

(UC = ulcerative colitis; $\mathrm{CAC}=$ colitis-associated cancer; $\mathrm{CRC}=$ colorectal cancer)

A.

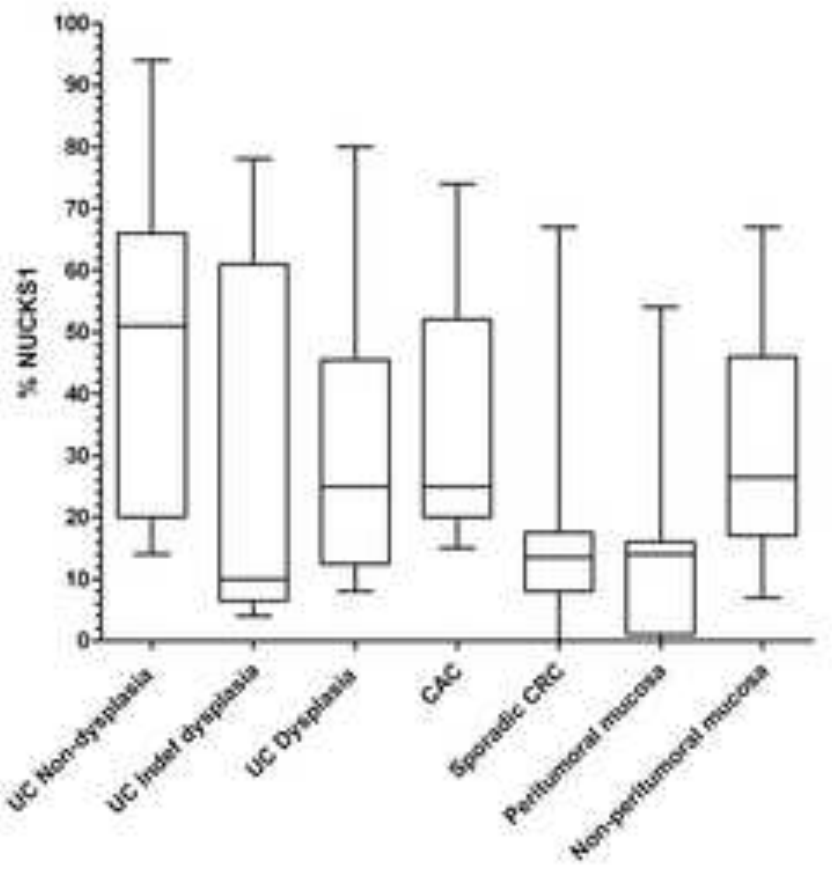

C.

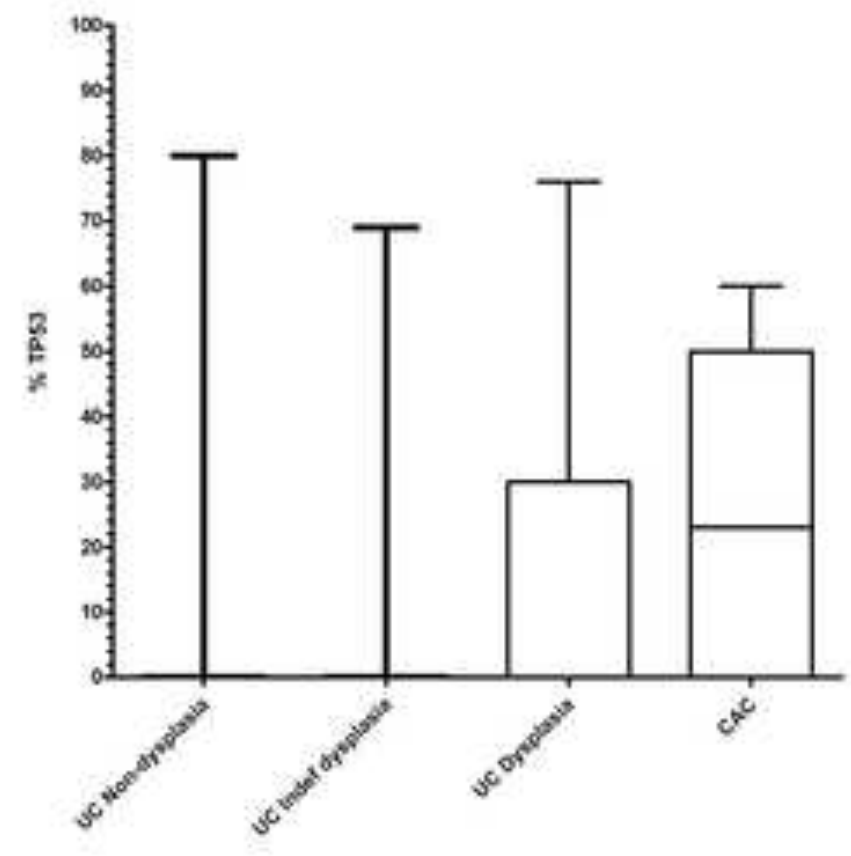

B.

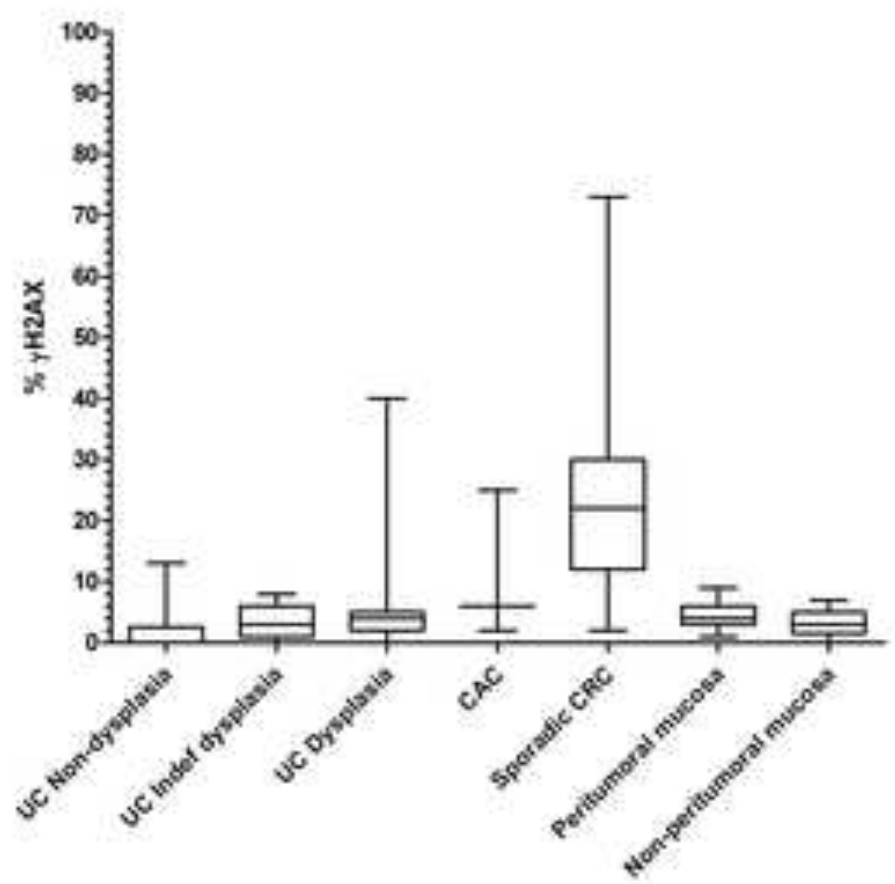

D.

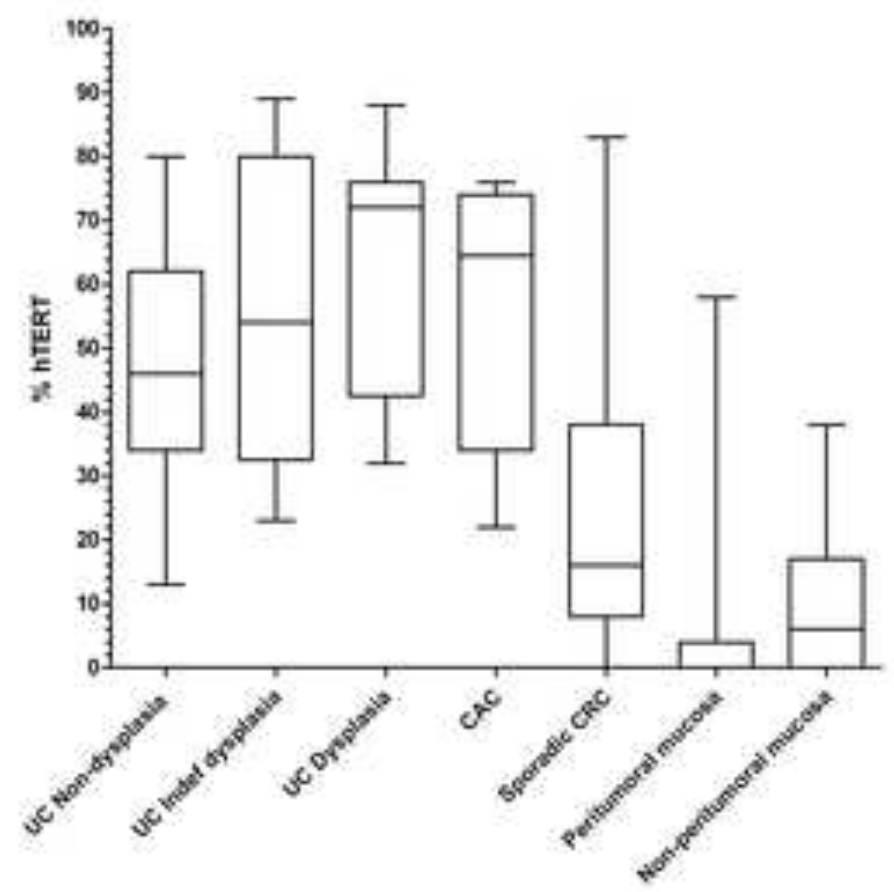

